\title{
Factors associated with dysfunction of autogenous arteriovenous fistula in patients with maintenance hemodialysis: a retrospective study
}

\author{
Yu Li", Wenhao Cui", Jukun Wang, Chao Zhang, Tao Luo \\ Department of General Surgery, Xuanwu Hospital, Capital Medical University, Beijing, China \\ Contributions: (I) Conception and design: T Luo, C Zhang; (II) Administrative support: T Luo, C Zhang; (III) Provision of study materials or \\ patients: Y Li; (IV) Collection and assembly of data: Y Li, W Cui, J Wang; (V) Data analysis and interpretation: Y Li, W Cui, J Wang, C Zhang; (VI) \\ Manuscript writing: All authors; (VII) Final approval of manuscript: All authors. \\ \#These authors contributed equally to this work. \\ Correspondence to: Tao Luo. Department of General Surgery, Xuanwu Hospital, Capital Medical University, No. 45, Changchun Street, Xicheng \\ District, Beijing, China. Email: TaoLuo35@126.com.
}

\begin{abstract}
Background: Patients in maintenance hemodialysis (MHD) need a patent vascular access for optimal treatment. Autologous arteriovenous fistula (AVF) is the optimal vascular access for patients with MHD. However, AVF dysfunction may limit its use. The aim of this article is to explore the factors associated with primary dysfunction of AVF in patients with MHD.

Methods: This is a retrospective study. A total of 121 patients who underwent anastomosis for AVF in our hospital from January $1^{\text {st }}, 2016$ through December $31^{\text {st }}, 2018$ were screened for eligibility. Of these patients, 44 were excluded according to the exclusion criteria. The remaining patients were divided into two groups based on the function of vascular access. The complete blood count and other blood biochemical parameters were compared between two groups. The risk factors associated with AVF dysfunction were analyzed by multivariate Cox proportional hazard regression model for patients with end-stage renal disease (ESRD).
\end{abstract}

Results: There were significant differences in serum phosphorus (P), total cholesterol (TC), low density lipoprotein $(\mathrm{LDL})$ and gender between patency and dysfunction groups of AVFs $(\mathrm{P}<0.05)$. Further multivariate COX proportional risk regression showed that hypercholesterolemia and hyperphosphatemia were independent risk factors for AVF dysfunction.

Conclusions: Hypercholesterolemia and hyperphosphatemia are independent risk factors for primary AVF dysfunction in patients with MHD.

Keywords: Autologous arteriovenous fistula (AVF); primary dysfunction; survival analysis; vascular access

Submitted Nov 06, 2020. Accepted for publication Feb 02, 2021.

doi: 10.21037/apm-20-2196

View this article at: http://dx.doi.org/10.21037/apm-20-2196

\section{Introduction}

Chronic kidney disease (CKD) is a condition of irreversible destruction of the renal parenchyma, with a progressive loss of kidney function over a period of several years. Currently, kidney transplantation is the most effective treatment for CKD. However, its application is limited because of the donor organ shortage. Thus, maintenance hemodialysis (MHD) becomes the best alternative for kidney transplant.
MHD patients require essential lifetime vascular access during hemodialysis. Autologous arteriovenous fistula (AVF) is the optimal vascular access for MHD. However, possible AVF dysfunction after surgery, particular for prolonged use, has limited its use. The patient had to undergo surgery again if the AVF could not meet the requirement. This will not only increase the costs, but also lead to the waste of vascular resources. Therefore, the purpose of this paper is to 
study the relevant factors associated with AVF dysfunction.

We present the following article in accordance with the STROBE reporting checklist (available at http://dx.doi. org/10.21037/apm-20-2196).

\section{Methods}

\section{Research object}

\section{Selection of study participants}

We selected 121 patients who underwent vascular anastomosis for MHD at the Department of Vascular Surgery, Xuanwu Hospital, Capital Medical University between January $1^{\text {st }}, 2016$ and December $31^{\text {st }}, 2018$.

\section{Inclusion criteria}

(I) Age $\geq 18$ years old at the time of fistula establishment; (II) patients who underwent AVF operation for the first time; (III) the newly established AVF was functioning normally, and the blood flow in AVF $\geq 250 \mathrm{~mL} / \mathrm{min}$; (IV) patients who underwent MHD in our hospital after AVF establishment; (V) patients who received surgery from the same doctor.

\section{Exclusion criteria}

(I) Patients unable to receive telephone follow-up for any reason; (II) patients with a short life expectancy due to various causes (e.g., tumors); (III) patients who underwent renal transplantation, were lost to follow-up, or died; (IV) patients with other causes of AVF dysfunction.

\section{Grouping of patients}

A total of 77 patients who met the inclusion criteria were included in the study. Patients were divided into patency $(n=47)$ and dysfunction $(n=30)$ groups based on whether their AVF was patent or not. The protocol of this retrospective study conformed to the ethical guidelines of the Declaration of Helsinki (as revised in 2013) and was approved by the Ethics Committee of Xuanwu Hospital \{No. L YS [2020]018\} with a waiver of informed consent.

\section{Research methods}

\section{Surgical procedure}

First, the necessity and safety of operation were carefully evaluated. Thereafter, the diameter of radial artery and cephalic vein for all patients were examined by ultrasound before operation. End to side forearm cephalic vein-radial artery anastomosis was performed in patients without contraindications under a local anesthesia. Ultrasounds for radial artery and cephalic vein were repeated after surgery, but before dialysis. Regular dialysis was started on nonstenosis patients. The operation was performed by the same doctor.

\section{Assessment for successful operation}

AVF operation was deemed to be successful if vascular thrill was palpable or auscultated, and the blood flow was above the minimum rate required for dialysis $(250 \mathrm{~mL} / \mathrm{min})$. A functional AVF must be absence of thrombosis, stenosis, hematoma, and other serious complications.

\section{Blood Biochemical tests}

The patients underwent routine fasting blood tests at least 3 months after initial dialysis. We collected the most recent data before AVF dysfunction (or before the study if AVF is patency). In addition, the blood samples were collected by experienced health professionals.

\section{Observation variables}

During the assessment period, we recorded the gender, age at the time of AVF establishment, BMI, primary disease of end-stage renal disease (ESRD), preoperative blood pressure, glycosylated hemoglobin (HbA1c), diameter of blood vessels, hemoglobin ( $\mathrm{Hb}$ ), albumin (ALB), total cholesterol (TC), total triglycerides (TT), low density lipoprotein (LDL), serum creatinine (Scr), and preoperative electrolyte condition (procalcitonin PTH, blood calcium, blood phosphorus). At the same time, AVF patency (blood flow $\geq 250 \mathrm{~mL} / \mathrm{min}$ ) was also recorded. There was no missing data in the study.

\section{Statistical analyses}

Continuous data were expressed as mean \pm standard deviation. Comparisons between groups were performed by independent $t$-tests if normal distribution is conformed. Mann Whitney $\mathrm{U}$ test was used if normal distribution was not conformed. Categorical variables were reported using frequency counts and percentages, and statistical differences between two groups were compared using chi-square test. First, univariate analysis was used to identify the variables with difference between patency and dysfunction group. Second, (multivariate) Cox proportional hazard regression was performed to further analyze the identified variables with differences. "Enter" method was adopted to screen variables. It was considered statistically significant if $\mathrm{P}<0.05$. Power test of the study was performed by PASS software. 
Meanwhile, statistical analyses were performed with SPSS 19.0.

\section{Results}

\section{Follow-up results and overall patency of fistula}

Generally, 121 patients with ESRD were initially assessed in this study. Of the 121 patients, 23 were lost to followup, 10 received kidney transplant, and 11 died; 77 patients who met the inclusion criteria were finally included in the analysis. Power of the data was 0.92 , which was included in the Cox model. Among them, 53 (68.8\%) were male. The mean age was $56.5 \pm 11.3$ years, ranging from 29 to 82 years old. Diabetic and non-diabetic nephropathy accounted for $47(61.0 \%)$ and $30(39.0 \%)$ of ESRD cases, respectively. All $\mathrm{AVF}$ s were matured, and the follow-up ranged from 5 to 39 months with a mean of $20.9 \pm 10.1$ months. Meanwhile, the follow-up period of patients in patency group and dysfunction group was $(19.7 \pm 9.9)$ months and $(22.8 \pm 10.2)$ months, respectively. Since the follow-up period was nonnormal distribution, Mann Whitney $\mathrm{U}$ test was performed. There was no statistically significant difference between the patency and dysfunction groups $(\mathrm{P}=0.191)$. During the observation period, $30(39.0 \%)$ patients developed AVF dysfunction whereas $47(61.0 \%)$ patients did not.

\section{Comparison of baseline between patency and dysfunction group}

The clinical and demographic characteristics of the entire study cohort are summarized in Table 1. A total of 20 study factors were included and compared between the patency and dysfunction group. $\mathrm{P}$ values of four variables were less than 0.1 (Screening variables), which were Gender, Phosphorus, TC and LDL. There was no significant difference for other factors (Table 1).

\section{AVF failure}

Figures 1-4 show the AVF patency survival curves with respect to $\mathrm{P}, \mathrm{TC}, \mathrm{LDL}$, and gender, respectively. Logrank tests indicated statistically significant differences between all 4 pairs of groups with $\mathrm{P}$ value of $0.007,0.001$, $0.012,0.013$, respectively. Previous studies have shown that HbA1c was higher in dysfunction group (1). In addition, age can affect the patency of AVFs (2). Therefore, P, TC, LDL, Gender, HbA1c and age were included in the multivariate Cox proportional hazard regression, which revealed that hypercholesterolemia and hyperphosphatemia were independent risk factors for AVF dysfunction $(\mathrm{P}<0.05)$ (Table 2).

\section{Discussion}

\section{Factors influencing development of AVF}

Over the past 5 decades, there has been a growing consensus of "fistula first" principle in patients with MHD. The guidelines, published in 1997 and revised in 2003 and 2006 (3-5), together with the "fistula first" principle proposed in 2003 (6), suggest that AVF is the optimal choice. Although AVF is associated with thrombosis, blood steal syndrome and aneurysm, incidence of these complications are relatively low for AVF. However, studies show that the AVF failure ranges between $15 \%$ and $22 \%(4,7)$. Recent studies suggested that the internal environmental factors are important for AVF success (8). Many factors caused by renal failure, such as high blood calcium, phosphorus and lipid, CRP, Scr, vascular diameter and preoperative radial artery calcification (RAC), may affect the patency of AVFs (9-12). Although some of these risk factors for AVF dysfunction have been well studied, many others remain elusive.

Since the "fistula first" principle in 2003, clinicians have increasingly preferred AVF for MHD (13). In this study, we found TC and Phosphorus to be the independent risk factors for AVF dysfunction.

\section{Age}

Most elderly patients present with other underlying diseases. As such, they may not harness optimal benefits from certain disease interventions, evidenced by short survival rate (14). In a separate but related study, it was found that the advantages of AVF over central venous catheter (CVC) gradually decreases with age (15). In contrast, the disadvantages of AVF are gradually amplified. As such, CVC usage may be preferred in elderly patients. Therefore, "fistula first" principle will face a challenge. Based on our study, age is not an independent risk factor for AVF dysfunction. Generally, age of patients does not affect the primary patency rate of AVF, but affect the long-term benefits. At the same time, from an economic point of view, AVF use for dialysis in elderly patients may be counterproductive. In a related study, Hall et al. established a Markov model from retrospective data 
Table 1 Baseline of patency and dysfunction group

\begin{tabular}{|c|c|c|c|c|}
\hline Characteristics & Overall $(n=77)$, mean \pm SD & Patency $(n=47)$, mean \pm SD & Dysfunction $(n=30)$, mean \pm SD & Sig \\
\hline Female (\%) & $24(31.2)$ & $11(45.8)$ & $13(54.2)$ & 0.066 \\
\hline Diabetic nephropathy (\%) & $47(61.0)$ & $29(61.7)$ & $18(38.3)$ & 0.881 \\
\hline Age (year) & $56.5 \pm 11.3$ & $55.6 \pm 12.2$ & $58.0 \pm 9.5$ & 0.799 \\
\hline $\mathrm{SBP}(\mathrm{mmHg})$ & $150.3 \pm 23.8$ & $149.5 \pm 23.0$ & $151.5 \pm 25.3$ & 0.657 \\
\hline $\mathrm{DBP}(\mathrm{mmHg})$ & $84.2 \pm 11.2$ & $84.6 \pm 10.4$ & $83.4 \pm 12.4$ & 0.775 \\
\hline $\mathrm{HbA1c}(\%)$ & $5.8 \pm 1.2$ & $5.8 \pm 0.9$ & $5.8 \pm 1.6$ & 0.607 \\
\hline PTH (pg/mL) & $259.4 \pm 192.8$ & $253.7 \pm 176.0$ & $268.4 \pm 219.3$ & 0.418 \\
\hline TT (mmol/L) & $1.7 \pm 1.0$ & $1.7 \pm 1.0$ & $1.8 \pm 1.1$ & 0.614 \\
\hline ALB (g/L) & $33.2 \pm 9.0$ & $33.4 \pm 10.5$ & $32.8 \pm 6.2$ & 0.496 \\
\hline $\operatorname{Scr}(\mu \mathrm{mol} / \mathrm{L})$ & $671.8 \pm 246.4$ & $649.6 \pm 239.6$ & $706.5 \pm 256.9$ & 0.326 \\
\hline $\mathrm{Ca}(\mathrm{mmol} / \mathrm{L})$ & $1.9 \pm 0.3$ & $2.0 \pm 0.3$ & $2.0 \pm 0.2$ & 0.929 \\
\hline $\mathrm{P}(\mathrm{mmol} / \mathrm{L})$ & $1.7 \pm 0.4$ & $1.6 \pm 0.4$ & $1.8 \pm 0.4$ & 0.004 \\
\hline CRP (mg/L) & $10.1 \pm 16.4$ & $10.1 \pm 17.2$ & $10.2 \pm 15.4$ & 0.807 \\
\hline Diameter of cephalic vein (mm) & $2.6 \pm 0.6$ & $2.6 \pm 0.5$ & $2.5 \pm 0.7$ & 0.764 \\
\hline Diameter of radial artery (mm) & $2.5 \pm 0.4$ & $2.6 \pm 0.3$ & $2.5 \pm 0.5$ & 0.392 \\
\hline
\end{tabular}

SBP, systolic blood pressure; DBP, diastolic blood pressure; HbA1c, glycosylated hemoglobin; PTH, parathyroid hormone; HB, hemoglobin; PLT, platelet; TT, total triglyceride; ALB, albumin; Scr, serum creatinine; Ca, calcium; P, phosphorus; CRP, C-reactive protein; TC, total cholesterol; LDL, low density lipoprotein.

to analyze the input-output ratio of patients with AVF, arteriovenous graft (AVG) and CVC (15). They found that the input-output of AVF against AVG and CVC gradually declines with patients' age. For the elderly Medicare patients in United States, the average annual cost of maintaining AVF patency for 2.5 years is $\$ 7,871$ whereas that of patients with primary dysfunction is $\$ 13,282$ per year (16). So, the benefits of AVF in elderly patients are suboptimal.

\section{Serum TC}

Blood lipid plays an important role in the patency of arteriovenous fistula. Previous study showed that TC was an independent risk factor for AVF dysfunction (17). Generally, high-density lipoprotein (HDL) is a protective factor for AVF patency. Wang et al. demonstrated that higher TT increases the risk of AVF dysfunction (18). However, based on our study, TC was an independent risk factor for AVF, but TT was not. This discrepancy may be attributed to the small sample size in our study. Hyperlipidemia can activate vascular endothelial growth factor $\mathrm{A} /$ matrix metalloproteinase-9 signaling pathway, which causes intimal hyperplasia and atherosclerosis (16).

\section{Blood phosphorus}

Emerging evidence indicated that blood phosphorus was 


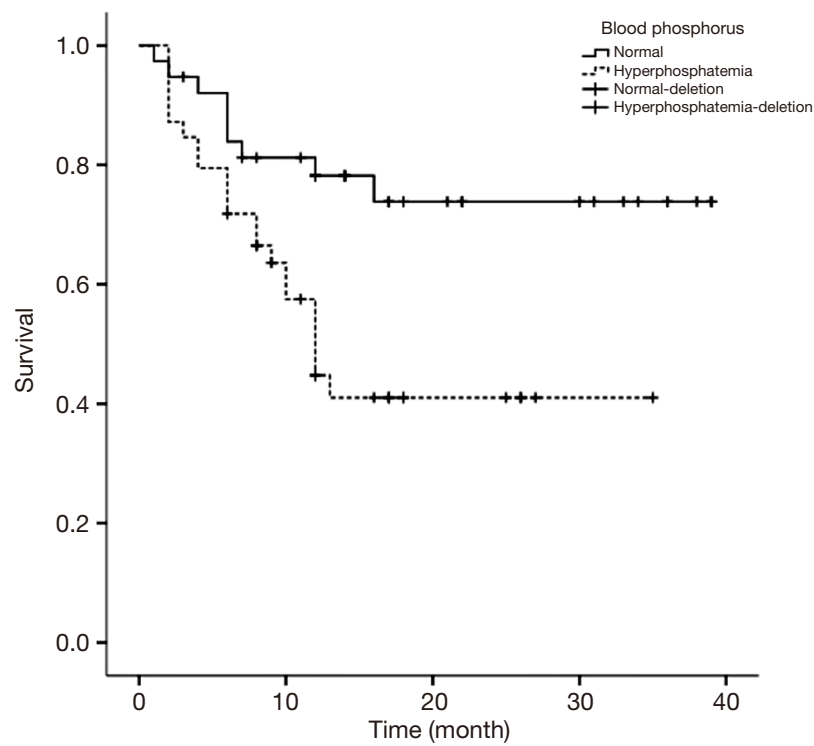

Figure 1 AVF survival curves for patients with hyperphosphatemia and normal blood phosphorus $(\mathrm{P}=0.007)$. AVF, autologous arteriovenous fistula.

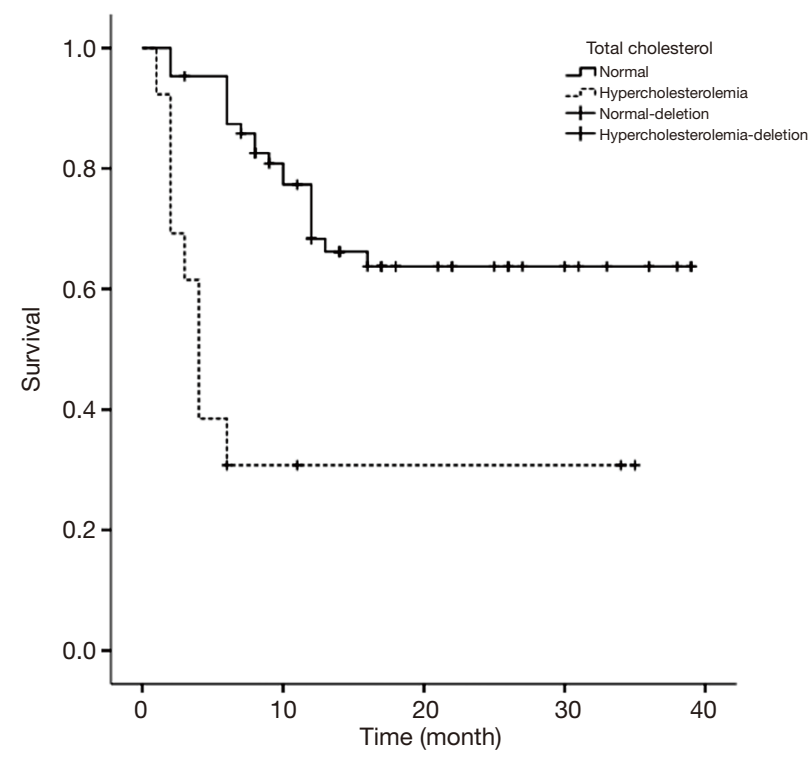

Figure 2 AVF survival curves for patients with hypercholesterolemia and normal TC $(\mathrm{P}=0.001)$. AVF, autologous arteriovenous fistula; TC, total cholesterol.

associated with AVF dysfunction. Moon and Lee showed that hyperphosphatemia (blood phosphorus $>5.5 \mathrm{mg} / \mathrm{mL}$ ) had a strong positive correlation with AVF dysfunction within 1 year of its induction (19). Consistent with

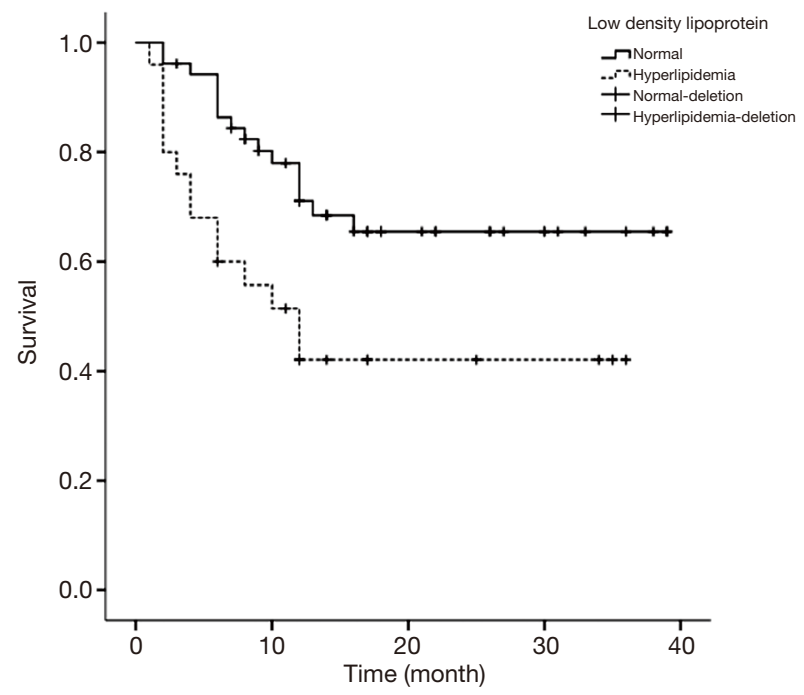

Figure 3 AVF survival curves for patients with increased LDL and normal LDL ( $\mathrm{P}=0.012)$. AVF, autologous arteriovenous fistula; LDL, low density lipoprotein.

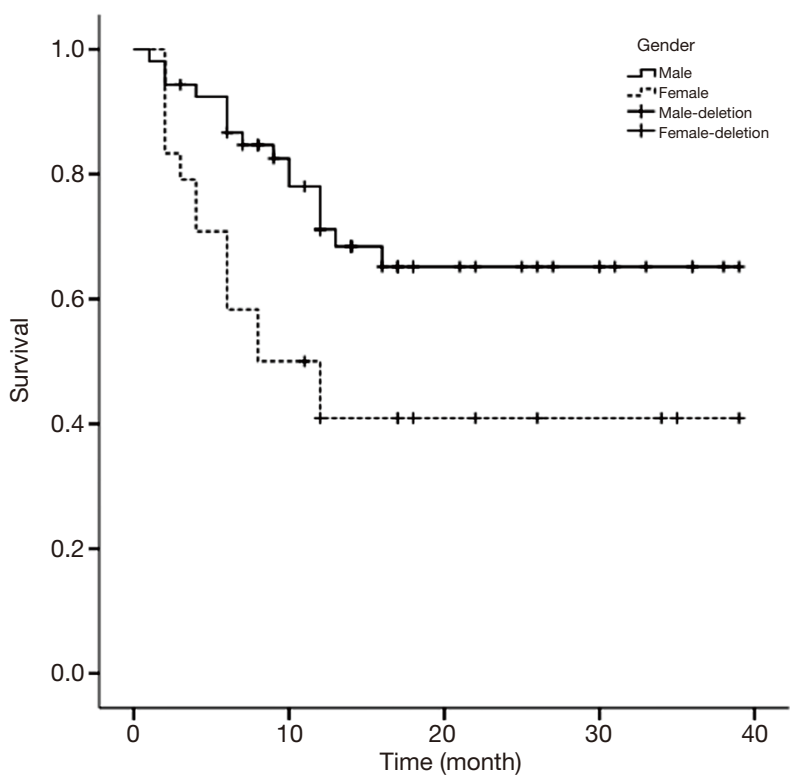

Figure 4 AVF survival curves for patients with male and female $(\mathrm{P}=0.013)$. AVF, autologous arteriovenous fistula.

our findings, Zhou et al. also found that patients with hyperphosphatemia were more likely to develop AVF dysfunction than their normal counterparts, indicating that hyperphosphatemia was an independent risk factor for AVF dysfunction (20). Continuous exploration of vascular access dysfunction has drawn interest in vascular calcification. 
Table 2 Multivariate cox proportional hazard regression analysis

\begin{tabular}{lccc}
\hline Risk factor & P value & RR value & $95 \% \mathrm{Cl}$ \\
\hline $\mathrm{P}$ & 0.032 & 2.39 & $1.08-5.25$ \\
TC & 0.043 & 3.12 & $1.04-9.36$ \\
LDL & 0.589 & 1.29 & $0.51-3.23$ \\
Gender & 0.278 & 1.57 & $0.69-3.57$ \\
HbA1c & 0.641 & 0.80 & $0.31-2.08$ \\
Age & 0.399 & 1.46 & $0.61-3.52$ \\
\hline
\end{tabular}

Blood phosphorus has been implicated for vascular calcification. Prolonged hyperphosphatemia can transform vascular smooth muscle cells (VSMC) into osteoblast like cells (21). This induces calcium and phosphorus deposition on the inner wall of blood vessels, thus causing vascular calcification.

\section{Scr}

Our result demonstrated that Scr is not an independent risk factor for AVF dysfunction, which is consistent with the previous study (22). Thus, the renal function (Scr) at the beginning of dialysis does not have a significant impact on the primary patency rate.

\section{Vessel diameter}

We also investigated the effect of vascular (arterial and venous) diameters on AVF primary dysfunction. Misskey et al. reported that radial arterial diameter $(<2.1 \mathrm{~mm})$ is an independent risk factor for AVF dysfunction (23). However, in this study, the result of Cox survival analysis didn't support preoperative vascular diameter to be an independent risk factor for AVF dysfunction, probably due to initial screening on vascular diameter before surgery at our center.

\section{Limitation}

We acknowledge that our study analyzes data from a single center and selection bias may be unavoidable. Being a retrospective study with a small sample size, we couldn't exclude other confounding. Due to the limitation of our dataset, 23 were lost to follow-up for various reasons, 10 received kidney transplant, and 11 died, which may lead to the deviation of the results. The small sample size might generate type- 1 and/or type-2 error. Although Power test was performed (Power $=0.92$ ), a larger sample size study is still needed in future. In addition, this is an exploratory study. The prospective cohort with larger sample size may be used to validate the risk factors, such as phosphorus and $\mathrm{TC}$, for AVF dysfunction in future.

\section{Conclusions}

Findings of this study implicated TC and phosphorus as independent risk factors for AVF dysfunction, and these factors can reduce the primary patency rate. Therefore, before the operation, patients should be assessed appropriately. Currently, there are many measures to correct hypercholesterolemia, among which the most commonly used are lipid-lowering drugs. And the outcome is satisfactory. However, it is relatively difficult to correct hyperphosphatemia. The main treatment measures were "3D principle", including diet, dialysis, and drugs (24). But the effect is unsatisfactory. Recently, we reported that high phosphorus can induce phenotype transformation of VSMC and eventually lead to intimal hyperplasia through DNA damage response pathway (25). Therefore, inhibitors targeting the relevant signaling pathway may block intimal hyperplasia, which may provide a new clue for the treatment of AVF dysfunction.

\section{Acknowledgments}

Funding: The study was supported by Beijing Hospitals Authority Youth Programme (QMS20200803 to CZ).

\section{Footnote}

Reporting Checklist: The authors have completed the STROBE reporting checklist. Available at http://dx.doi. org/10.21037/apm-20-2196

Data Sharing Statement: Available at http://dx.doi. org/10.21037/apm-20-2196

Peer Review File: Available at http://dx.doi.org/10.21037/ apm-20-2196

Conflicts of Interest: All authors have completed the ICMJE uniform disclosure form (available at http://dx.doi. org/10.21037/apm-20-2196). The authors have no conflicts of interest to declare. 
Ethical Statement: The authors are accountable for all aspects of the work in ensuring that questions related to the accuracy or integrity of any part of the work are appropriately investigated and resolved. The study protocol conformed to the ethical guidelines of the Declaration of Helsinki (as revised in 2013), and the Ethics Committee of Xuanwu Hospital approved this study \{No. L YS [2020]018\}. Because of the retrospective nature of the research, the requirement for informed consent was waived.

Open Access Statement: This is an Open Access article distributed in accordance with the Creative Commons Attribution-NonCommercial-NoDerivs 4.0 International License (CC BY-NC-ND 4.0), which permits the noncommercial replication and distribution of the article with the strict proviso that no changes or edits are made and the original work is properly cited (including links to both the formal publication through the relevant DOI and the license). See: https://creativecommons.org/licenses/by-nc-nd/4.0/.

\section{References}

1. Wärme A, Hadimeri $\mathrm{H}, \mathrm{Nasic} \mathrm{S}$, et al. The association of erythropoietin-stimulating agents and increased risk for AV-fistula dysfunction in hemodialysis patients. A retrospective analysis. BMC Nephrol 2021;22:30.

2. Woo K, Lok CE. New Insights into dialysis vascular access: What is the optimal vascular access type and timing of access creation in CKD and dialysis patients? Clin J Am Soc Nephrol 2016;11:1487-94.

3. NKF-DOQI clinical practice guidelines for vascular access. National Kidney Foundation-Dialysis Outcomes Quality Initiative. Am J Kidney Dis 1997;30:S150-91.

4. NKF-K/DOQI Clinical Practice Guidelines for Vascular Access: update 2000. Am J Kidney Dis 2001;37:S137-81.

5. Vascular Access Work Group. Clinical practice guidelines for vascular access. Am J Kidney Dis 2006;48 Suppl 1:S248-73.

6. Hemodialysis Adequacy 2006 Work Group. Clinical practice guidelines for hemodialysis adequacy, update 2006. Am J Kidney Dis 2006;48 Suppl 1:S2-90.

7. C Ren, $W$ Lu, X Yang, et al. Analysis on the application and the related factors of vascular access at initiation of hemodialysis in Shanghai. Chinese Journal of Blood Purification 2016;15:344-7.

8. Khavanin Zadeh M, Mohammadipour S, Omrani Z. Correlation between CRP and early failure of arteriovenous fistula (AVF). Med J Islam Repub Iran
2015;29:219.

9. Khavanin Zadeh M, Gholipour F, Naderpour Z, et al. Relationship between vessel diameter and time to maturation of arteriovenous fistula for hemodialysis Access. Int J Nephrol 2012;2012:942950.

10. Chen Z, Zeng H, Huang F, et al. Effect of radial artery calcification on survival of arteriovenous fistula and the patients in end-stage renal disease patients. China Medical Abstracts (Internal Medicine) 2019;36:52.

11. National Kidney Foundation (2015). KDOQI Clinical Practice Guideline for Hemodialysis Adequacy: 2015 update. Am J Kidney Dis 2015;66:884-930.

12. He C, Zhang X. Renal replacement therapy. Shanghai: Science and Technology Education Press, 2005:152-4.

13. Wen $\mathrm{M}, \mathrm{Li} Z, \mathrm{Li}$ J, et al. Risk factors for primary arteriovenous fistula dysfunction in hemodialysis patients: A retrospective survival analysis in multiple medical centers. Blood Purif 2019;48:276-82.

14. Lee T, Allon M. Reassessing Recommendations for Choice of Vascular Access. Clin J Am Soc Nephrol 2017;12:865-7.

15. Hall RK, Myers ER, Rosas SE, et al. Choice of hemodialysis access in older adults: A cost-effectiveness analysis. Clin J Am Soc Nephrol 2017;12:947-54.

16. Thamer M, Lee TC, Wasse H, et al. Medicare Costs Associated With Arteriovenous Fistulas Among US Hemodialysis Patients. Am J Kidney Dis 2018;72:10-8.

17. Cui TL, Zhang R, Liu F, et al. Effect of diabetes mellitus on early dysfunction of arteriovenous fistula in patients with end-stage renal disease. Sichuan Da Xue Xue Bao Yi Xue Ban 2012;43:438-41.

18. Li Z, Wang W, Jiang Y, et al. Analysis of influencing factors of failure of autogenous arteriovenous fistula in maintenance hemodialysis patients. Chinese Journal of Integrated Traditional and Western Nephrology 2014;15:961-4.

19. Moon JY, Lee HM, Lee SH, et al. Hyperphosphatemia is associated with patency loss of arteriovenous fistula after 1 year of hemodialysis. Kidney Res Clin Pract 2015;34:41-6.

20. Zhou M, Lu F. Effect of hyperphosphatemia on patency rate after reoperation of autogenous arteriovenous fistula dysfunction. Chinese Journal of Medicine 2018;98:3406-10.

21. Shimokado A, Sun Y, Nakanishi M, et al. Smad3 plays an inhibitory role in phosphate-induced vascular smooth muscle cell calcification. Exp Mol Pathol 2014;97:458-64.

22. Stolic RV, Trajkovic GZ, Kostic MM, et al. Factors affecting the patency of arteriovenous fistulas for hemodialysis: Single center 307 experience. Hemodial Int 
2018;22:328-34.

23. Misskey J, Hamidizadeh R, Faulds J, et al. Influence of artery and vein diameters on autogenous arteriovenous access patency. J Vasc Surg 2020;71:158-72.e1.

24. Chen $\mathrm{W}, \mathrm{Yu} X$. Challenges and advances in the management of hyperphosphatemia in dialysis patients with chronic kidney disease. Chinese Journal of Nephrology 2018;34: 867-71.

25. Hénaut L, Mary A, Chillon JM, et al. The Impact of Uremic Toxins on Vascular Smooth Muscle Cell Function. Toxins (Basel) 2018;10:218.

Cite this article as: Li Y, Cui W, Wang J, Zhang C, Luo T. Factors associated with dysfunction of autogenous arteriovenous fistula in patients with maintenance hemodialysis: a retrospective study. Ann Palliat Med 2021;10(4):4047-4054. doi: 10.21037/apm-20-2196 